\title{
Gastrointestinal Motility: An Academic and Research Perspective
}

\author{
Eamonn M.M. Quigley \\ Medical School, National University of Ireland, Cork, Ireland
}

\section{Key Words \\ Gastrointestinal motility · Gastrointestinal motility research, research opportunities . Neurogastroenterology $\cdot$ Gastroenterology $\cdot$ Smooth muscle $\cdot$ Enteric nervous system}

\begin{abstract}
While, in the past, gastrointestinal motility may have been viewed as a narrow and restricted field, confined to the study of twitches in muscle baths and squiggles on smoke drums, it has, of late and belatedly, entered into the mainstream of gastroenterology and medicine. As a consequence, this field, now more correctly and appropriately described as neurogastroenterology, concerns itself with a vast spectrum of clinical disorders of varying pathophysiology, presentation and management. Never before has this area offered so many opportunities to the budding academician and researcher.
\end{abstract}

Copyright (c) 2006 S. Karger AG, Basel

Any discussion of gastrointestinal motility must begin with some attempt to define this area of clinical and research endeavor which, though close to the hearts of those of us who toil in this field, means little or nothing to many of our medical colleagues, not to mind the lay public.

In the past, motility was viewed narrowly as that field of medicine which dealt with disorders of motor function in the gastrointestinal tract. The focus of both research and clinical studies was on the movement of contents along the gut and included studies of swallowing, gastric emptying, small bowel and colonic transit and defecation. With the advent of manometry, the contractile contributors to these phenomena could be studied and much attention was focused on the esophagus, lower esophageal sphincter and the ano-rectum. As a consequence of the application of these methodologies to patient populations, such disorders as achalasia, gastroparesis, intestinal pseudo-obstruction, idiopathic megacolon and Hirschsprung's disease were described and the contributions of motor dysfunction to gastroesophageal reflux disease (GERD), diverticulosis, constipation and incontinence explored.

With the advent of an appreciation of the sensory function of the gut, as well as the complexity of brain-gut interactions and the potential for disease at any level, from the mucosal sensory receptor, through the spinal cord and brain, along the autonomic nervous system and right down to gut smooth muscle, to lead to symptoms, the narrow focus of motility as a concept and as a discipline has been revised. This discipline, which some would rather refer to as neurogastroenterology [1], is now more accurately defined as the study of gastrointestinal muscle and nerve, their regulation and their integration with gastrointestinal function as well as with the central nervous and neuroendocrine systems.

At the same time, we have come to appreciate the prevalence and clinical importance of gut motor dysfunction in systemic disorders, such as neurological [2], skeletal muscle and connective tissue disorders, as well as in the critically ill $[3,4]$. Motilists have latterly strayed into the

\section{KARGER}

Fax +4161306 1234 E-Mail karger@karger.ch www.karger.com
(C) 2006 S. Karger AG, Basel

$0257-2753 / 06 / 0244-0218 \$ 23.50 / 0$

Accessible online at: www.karger.com/ddi
Eamonn M.M. Quigley, MD

Alimentary Pharmabiotic Centre, Department of Medicine

Clinical Sciences Building, Cork University Hospital

Cork (Ireland)

Tel. +353 21490 1228, Fax 35321490 1289, E-Mail e.quigley@ucc.ie 
functional disorders where symptoms may mimic those of more clearly defined motor disorders and where prior manometric studies suggested a role for motor dysfunction. This foray has not necessarily helped to improve the perception of the motilist. Now more than ever, the laborer in this vineyard is seen to be dealing with the interpretation of vague and non-specific symptoms and in attempting to make sense of 'motility' disorders, many of whom remain poorly defined. As a consequence of dabbling in irritable bowel and related disorders, his or her tests are viewed as poorly validated and their role in diagnosis and planning of management unclear. Furthermore, and despite considerable advances in our understanding of gut muscle, the enteric nervous system and the gut-brain axis, there are few and relatively ineffective therapies in the armamentarium. As a consequence, motility is perceived as being unattractive as a career option, training, in this area, is usually inadequate and often nonexistent and few are encouraged to pursue research in motility and neurogastroenterology. In summary, motility has a real image problem.

\section{The Way Forward}

Rather than pursue endless arguments on the role of motility, motility testing and prokinetic therapy in the functional gastrointestinal disorders, the motility expert should revert to first principles and learn all that one can about relationships between symptoms, dysfunction and pathology from well-defined disorders of intestinal nerve and muscle [5]. Here I am referring to achalasia, Chagas' disease, scleroderma and Hirschsprung's disease, in particular. Here we understand much of the pathophysiology and, in some instances, of the pathogenesis of the disease. We can carefully track symptom evolution and correlate scintigraphic or manometric abnormalities with pathology. We also need to pay much more attention to the motility problems which afflict the critically ill and which have come to represent a major issue for all of us who work in a busy acute hospital. Here I am referring to such conditions as gastroparesis, ileus and Ogilvie's syndrome which may afflict the patients who people our intensive care units and whose motility problems may threaten not only their nutritional status, but also their very survival. Here there is not only a great need for expert clinical skills but an enormous opportunity for really relevant clinical research. Motility problems loom large for the many patients afflicted by neurological and muscle diseases, yet these problems have received scant attention in the med- ical literature. The potential to link a clinical presentation to a specific neurological lesion or muscle pathology should surely attract more interest, especially, in an era of advanced brain imaging. I hope that the next decades will see a re-orientation of the field to take account of what we know, understand what disorders may truly reflect enteric neuromuscular dysfunction and concentrate on the validation of our tests and therapies in appropriate clinical contexts. I see the motilist of the future as a truly renaissance individual whose practice is based on a thorough understanding of motor physiology, including that of the gut-brain axis, who has a full appreciation of the range of disorders truly associated with motor dysfunction, enjoys a wealth of experience in the performance and understanding of all tests relevant to the assessment of gastrointestinal motor dysfunction (including imaging and endoscopic techniques) and has real expertise not only in the management of gastrointestinal motor dysfunction and its consequences, including the pharmacological treatment of symptoms, but also in addressing the psychosocial and nutritional problems which frequently complicate these disorders.

\section{Research Opportunities in Gastrointestinal Motility}

There has never been a greater need, or a greater opportunity, for research in the area of gastrointestinal motility. Given the issues that have been discussed above, it should be self-evident that the field is literally wide open for the application of new ideas and approaches. Opportunities abound in all types of research.

In epidemiology, there is the potential to define the true spectrum of motility disorders and especially in systemic disease, such as in the ICU patient, in liver and neurological disease. Epidemiological studies have latterly defined the true prevalence of many functional disorders; there is much to earn from further studies of the associations between these disorders, per se, as well as their constituent sub-groups, and other diseases as well as various demographic features. The application of the new field of molecular epidemiology to irritable bowel syndrome (IBS) has already produced some intriguing findings; there is so much more to learn? Why, for example, do some individuals develop IBS following a single episode of bacterial gastroenteritis yet the majority do not?

The application of consensus definitions to the descriptions of functional disorders has facilitated multinational epidemiological and therapeutic studies [6]; the 
stage is now set for a thorough assessment of the accuracy and appropriateness of these definitions to various cultures and populations. There is much to be learnt from the manner in which these disorders present and are managed across the globe [7]. While other areas of medicine have gained much from the rigorous application of the methods of evidence-based medicine, motility and functional disorders have been relatively unscathed. The time is surely ripe for a true outcome assessment of manometric and transit studies in gastroenterology. More work is also needed to produce validated instruments for the assessment of symptoms and quality of life, as well as a more complete understanding of their psycho-social dimensions. Our understanding of some of our most common and challenging conditions, such as functional heartburn, idiopathic gastroparesis, 'severe' IBS (where does IBS end and pseudo-obstruction begin?), ileus, megacolon and so-called 'obstructed' defecation is minimal, at any level; they all deserve the intense scrutiny of the clinical and basic science researcher, alike.

The advent of molecular biology has already yielded much in an area where many would have expected little. The unraveling of the genetic basis for Hirschsprung's disease [8] and some varieties of chronic idiopathic intestinal pseudo-obstruction has also provided invaluable insights into the development of the enteric nervous system itself. We are now in an era where the genetic contributions to IBS and GERD are being dissected and techniques of molecular pharmacology are being applied to the management of IBS.

Those interested in new diagnostic methods have many new toys: What will impedance tell us of the contributions of non-acid reflux to GERD? Will the barostat or tensostat finally permit an accurate assessment of in- testinal tone? What new information on contractile patterns and their relationship with transit will be provided by computerized multi-channel manometry? Have we only begun to see the potential of magnetic resonance imaging in the assessment of motor function?

At a more pathophysiological level, we need much more information on the integration of the central and enteric nervous systems and their respective roles in disease. In motility we have suffered greatly from a lack of clinical-pathological correlates; studies revealing deficiencies of interstitial cells of Cajal in disease states [9, 10] illustrate the potential of this field of research which continues to retain Cinderella status. We need new ideas here!

What is the role of inflammation in motor and functional disorders [11]? Here is a question that would have sounded ridiculous just a few years ago yet whose solution provides a tremendous opportunity for cross-disciplinary investigation involving clinicians, and basic scientists from fields as diverse as genetics, microbiology, immunology and pathology.

To enthuse a new generation about motility research we need to get them to think 'outside the box' and to look on this field afresh. We need to convince them that motility has emerged from the restricted confines of "traditional' physiology and pharmacology, has left behind it the limitations of a discipline whose intellectual exertions were confined to the subjective interpretation of squiggles on a smoke drum and has entered the new world of evidence-based, whole-body medicine where molecular biology and advanced technology are applied and rigorously assessed and where the potential for truly clinically meaningful progress is limitless.

\section{References}

$\checkmark 1$ Wingate DL, Grundy D: Neurogastroenterology and motility: at last, an equal partnership. Neurogastroenterol Motil 2000;12:1.

2 Quigley EMM, Pfeiffer RF (eds): Neurogastroenterology - gastrointestinal dysfunction in neurological disease. London, Butterworths, 2004.

3 Quigley EMM: Gastrointestinal motility disorders in severely ill patients. Turk J Gastroenterol 2002;13(suppl 2):39-44.

4 Quigley EMM: Critical care dysmotility: abnormal foregut motor function in the ICU/ITU patient. Gut 2005;54:1351-1352.
-5 Quigley EMM: Enteric neuropathology - recent advances and implications for clinical practice. Gastroenterologist 1997;5:233-241.

6 Drossman DA, Corazziari E, Talley NJ, Thompson WG, Whitehead WE (eds): Rome II. The functional gastrointestinal disorders: diagnosis, pathophysiology, and treatment: a multinational consensus. Virginia, Degnon Associates, 2000, pp 91-156.

7 Kang KY: Systematic review: the influence of geography and ethnicity in irritable bowel syndrome. Aliment Pharmacol Ther 2005;21: 663-676.

8 Milla PJ: Endothelins, pseudo-obstruction and Hirschsprung's disease. Gut 1998;44:148152.
-9 Zarate N, Mearin F, Wang XY, et al: Severe idiopathic gastroparesis due to neuronal and interstitial cells of Cajal degeneration: pathological findings and management. Gut 2003; 52:966-970.

10 Pardi DS, Miller SM, Miller DL, et al: Paraneoplastic dysmotility: loss of interstitial cells of Cajal. Am J Gastroenterol 2002;97:18281833.

11 Quigley EMM: Irritable bowel syndrome and inflammatory bowel disease: interrelated diseases? Chin J Dig Dis 2005;6:122-132. 